\title{
Isolation and Identification of PPFM Bacterial Isolates Isolated From Direct Seeded Rice Growing Areas of Hyderabad-Karnataka, India
}

\author{
J. Raghavendra $^{1 *}$, G.P. Santhosh ${ }^{1}$, Mahadevaswamy ${ }^{1}$, S. Shubha ${ }^{1}$ and M.V. Ravi ${ }^{2}$ \\ ${ }^{1}$ Department of Agriculture Microbiology, ${ }^{2}$ Department of Soil Science and Agricultural \\ Chemistry, University of Agriculture Sciences, Raichur-585104, Karnataka, India \\ *Corresponding author
}

\section{A B S T R A C T}

\section{Keywords}

PPFM's, Direct

seeded rice,

Rhizosphere,

Phyllosphere and

Methylobacterium

Article Info

Accepted:

04 June 2019

Available Online:

10 July 2019
A total of 50 PPFM's were isolated from rhizosphere soil and phyllosphere of direct seeded rice regions of Hyderabad-Karnataka. Samples of leaves and rhizosphere soils were collected from direct seeded rice grown in different location (Raichur district, Bellary district, Koppal district and Yadgir district). Thereafter, all the classical recommended methods used for identification of all the obtained isolates were carried out including, morphological, microscopical as well as biochemical characteristics. The results revealed that all the isolates were gram negative, rod shaped, motile. Colonies were pink, pale pink or dark pink in color due to pigmentation. Biochemical characteristics revealed that all the isolates including reference strain confirmed to be negative for Methyl red and VogesProskauer test while, positive for oxidase, urease, catalase activity, indole production and citrate utilization. None of the isolates could reduce nitrate to nitrite, whereas; the hydrolysis of casein and starch was not recorded in any of the isolates tested They can be cultivated on ammonium mineral salt (AMS) agar with methanol colonies were pink, pale pink or dark pink in color due to pigmentation and variations in colonial morphology. Based on the observed characteristics, the isolates obtained belong to the genus Methylobacterium.

\section{Introduction}

Rice is a staple food grown in almost all states of India accounting for more than 40 per cent of the food grain production. It is manually transplanted in standing water, destroys soil structure, is labour intensive and cumbersome. Hence, direct seeded rice without puddling is a viable alternative for its establishment as it can save water and also requires less labour. Methylotrophic bacteria are successful example that can achieve the two purposes as PGPR with antifungal activity. Many of the microbes living on the phylloplane probably lead a saprophytic lifestyle, feeding on materials leached from the leaf. One such 
example is Methylobacterium sp. a pink pigmented facultative methylotroph (PPFM's) which was first identified as covert contaminants from the tissue culture of liverwort, scapania nemorosa (Basile et al., 1969). This bacterium provides a useful model for the unappreciated kinds of interactions between plants and bacteria that take place routinely on lab and in culture dishes (Green and Bousifield, 1982).

The genus Methylobacterium is composed of a variety of pink pigmented methylotroph (PPFM) bacteria which are capable of growing on $\mathrm{C}_{1}$ compounds such as formate, formaldehyde, methanol and methylamine as well as on a wide range of multicarbon growth substates such as $\mathrm{C}_{2}, \mathrm{C}_{3}$ and $\mathrm{C}_{4}$ compounds. PPFMs are ubiquitous in nature and frequently reported on various plant species, those are a substantial part of the aerobic, heterotrophic microflora of the surfaces of young leaves. These bacteria are commonly found in soils, as well as on the surfaces of leaves, seeds and in the rhizosphere of a wide variety of plants, with highest numbers on actively growing and meristamatic tissue (Holland, 1997) Methylotrophs have been reported to influence seed germination and seedling growth by producing plant growth regulators like zeatin and related cytokinins and auxins and to alter agronomic traits like branching, seedling vigour, rooting and heat/cold tolerance (Omer et al., 2004).

Members of the genus Methylobacterium are pink-pigmented facultative methylotrophs (PPFMs). They belong to the proteobacterial sub group (class) Alpha-proteobacteria, order Rhizobiales, and family Methylobacteriaceae. They are strictly aerobic, Gram-negative and rod shaped. Methylobacterium are found worldwide on the leaves of many different plant species. The unique feature of PPFM's is their ability to oxidize methanol, a methylotrophic property based on the presence of methanol dehydrogenase (mxaF) gene.

\section{Materials and Methods}

Isolation and purification of PPFMs isolates

A detailed survey was conducted in different locations of Hyderabad- Karnataka for isolation of an efficient strain of PPFM. Samples of leaves and rhizosphere soils were collected from direct seeded rice grown in different villages such as UASR Agriculture farm, Kasbali camp, Kappagal, Nir Manvi and Sindhanur from Raichur district, Dadesuguru and Siriguppa from Bellary district, Gangavati and Karatagi from Koppal district, Hunasagi, Shahapur, and Balichakra from Yadgir district.

The samples were brought to the laboratory in sterile polythene bags and stored at $4^{\circ} \mathrm{C}$ to isolate methylotrophs.

About 50 different PPFM isolates from the rhizosphere and phyllosphere of direct seeded rice. Ammonium Mineral Salts (AMS) medium (Whittenbury et al., 1970) was used as a selective medium for isolation of PPFMs.

The AMS medium was sterilized by autoclaving at $121^{\circ} \mathrm{C}$ for 20 minutes and cooled to $50^{\circ} \mathrm{C}$. Filter sterilized methanol was added @ 0.5 per cent (v/v) after sterilization and before pouring media on to Petri plates. To isolate the PPFM bacteria from Phyllosphere region cyclohexamide $(20 \mu \mathrm{g} / \mathrm{ml})$ was added to the medium to avoid the fungal contamination. The $\mathrm{pH}$ of the medium was adjusted to 6.7 .

\section{Leaf imprinting technique}

On the solidified AMS agar medium upper and lower surface of leaf samples were placed separately, in such a way to impression on it. Then the leaves were lifted away and plates were incubated at $30{ }^{\circ} \mathrm{C}$ for 5-7 days (Corpe et al., 1985). 


\section{Serial dilution technique}

One gram sample of rhizosphere soil and leaves was ground using a pestle and mortar, serially diluted up to $10^{-6}$ dilutions and one $\mathrm{ml}$ each of the aliquots from $10^{-4}, 10^{-5}$ and $10^{-6}$ dilutions was transferred to sterile Petri dishes. The AMS medium was sterilized by autoclaving at $121^{\circ} \mathrm{C}$ for $20 \mathrm{~min}$ and cooled to $50{ }^{\circ} \mathrm{C}$. Filter sterilized cyclohexamide $(30 \mu \mathrm{g}$ $\mathrm{ml}^{-1}$ ) antibiotic solution and 0.5 per cent (v/v) methanol were incorporated into the sterilized AMS medium before plating. After plating with AMS medium, the plates were incubated in an inverted position for 5-7 days at $30^{\circ} \mathrm{C}$. Characteristic pink colonies growing over the medium were identified (Corpe and Rheem, 1989). Further, the methylotrophs were purified by the streak plate method and well isolated colonies on the plates were preserved on AMS agar slants. Isolates were maintained on AMS slants at $4{ }^{\circ} \mathrm{C}$ in a refrigerator for further use.

\section{Morphological and biochemical characterization}

Morphological and biochemical characterization will be carried out as per the standard procedures for the selected rhizosphere and phyllosphere group.

\section{Results and Discussion}

\section{Isolation of pink pigmented facultative methylotrophs from direct seeded rice}

In the present study, 50 PPFM's were isolated from rhizosphere soil and phyllosphere of direct seeded rice regions of HyderabadKarnataka. Out of fifty isolates, 25 were isolated from rhizosphere soil while remaining 25 isolates were isolated from phyllosphere of direct seeded rice. All the isolates were isolated by leaf imprinting and serial dilution method using AMS medium which was a selective medium for isolating PPFM's (Lidstrom and Chistoserdova, 2002). Several authors have already reported the natural association of PPFM's with several plants. Basile et al., (1969) for the first time reported these organisms as a contaminant of tissue cultures of the leafy liverwort, Scapania nemorosa which lead to conclude that these organisms as inhabitants of plant surface. They acted as phytosymbionts by associating with roots, leaves and seeds of most terrestrial plants (Trotsenko et al., 2001; Radha et al., 2007). The isolation of many PPFM's on the phyllosphere of plants has been reported by Kouno and Ozoki (1975). Among different crop plants, their relationships with the cereals have been reported by Balachandra et al., (2008), Kim et al., (2010) and Madhaiyan et al., (2003).

\section{Morphological and Biochemical characterization of PPFM isolates}

The results on characterization of PPFM isolates isolated from direct seeded rice, revealed that all the fifty PPFM isolates including reference strain were rod shaped, stained Gram negative and exhibited motility with accumulation to produce poly $\beta$-hydroxy butyrate granules. The observations were consistent with the report of Green and Bousifield (1982).

All PPFM isolates, obtained in the present study, exhibited pink colored colonies on ammonium mineral salts medium (AMS) with varying intensities of pigmentation ranging from light to dark pink which makes them to differentiate from other distinct methylotrophic organisms which are generally existing on plant surfaces.

These observations were confirmative with the reports of Corpe and Basile (1982) who observed that PPFM's were distinctive pink pigmentation due to carotenoids. 
Table.1 Sources of PPFM isolates from major direct seeded rice growing areas of Hyderabad-Karnataka region

\begin{tabular}{|c|c|c|c|}
\hline Sl. No. & Isolate code & Habitat & Place \\
\hline \multicolumn{4}{|c|}{ Raichur district } \\
\hline 1 & PPFM-1 & Rhizosphere & Kasbe Camp \\
\hline 2 & PPFM-2 & Rhizosphere & Kasbe Camp \\
\hline 3 & PPFM-3 & Phyllosphere & Kasbe Camp \\
\hline 4 & PPFM -4 & Phyllosphere & Kasbe Camp \\
\hline 5 & PPFM-5 & Rhizosphere & UASR Agriculture farm \\
\hline 6 & PPFM-6 & Rhizosphere & UASR Agriculture farm \\
\hline 7 & PPFM-7 & Phyllosphere & UASR Agriculture farm \\
\hline 8 & PPFM-8 & Phyllosphere & UASR Agriculture farm \\
\hline 9 & PPFM-9 & Rhizosphere & Kappagal \\
\hline 10 & PPFM-10 & Rhizosphere & Kappagal \\
\hline 11 & PPFM-11 & Phyllosphere & Kappagal \\
\hline 12 & PPFM-12 & Phyllosphere & Kappagal \\
\hline 13 & PPFM-13 & Rhizosphere & Sindhanur \\
\hline 14 & PPFM-14 & Rhizosphere & Sindhanur \\
\hline 15 & PPFM-15 & Phyllosphere & Sindhanur \\
\hline 16 & PPFM-16 & Phyllosphere & Sindhanur \\
\hline 17 & PPFM-17 & Rhizosphere & Nir manvi \\
\hline 18 & PPFM-18 & Rhizosphere & Nir manvi \\
\hline 19 & PPFM-19 & Phyllosphere & Nir manvi \\
\hline 20 & PPFM-20 & Phyllosphere & Nir manvi \\
\hline \multicolumn{4}{|c|}{ Bellary district } \\
\hline 21 & PPFM-21 & Rhizosphere & Dadesuguru \\
\hline 22 & PPFM-22 & Rhizosphere & Dadesuguru \\
\hline 23 & PPFM-23 & Phyllosphere & Dadesuguru \\
\hline 24 & PPFM-24 & Phyllosphere & Dadesuguru \\
\hline 25 & PPFM-25 & Rhizosphere & Siriguppa \\
\hline 26 & PPFM-26 & Rhizosphere & Siriguppa \\
\hline 27 & PPFM-27 & Phyllosphere & Siriguppa \\
\hline 28 & PPFM-28 & Phyllosphere & Siriguppa \\
\hline \multicolumn{4}{|c|}{ Koppal District } \\
\hline 29 & PPFM-29 & Rhizosphere & Gangavati ARS Farm \\
\hline 30 & PPFM-30 & Rhizosphere & Gangavati ARS Farm \\
\hline 31 & PPFM-31 & Phyllosphere & Gangavati ARS Farm \\
\hline 32 & PPFM-32 & Phyllosphere & Gangavati ARS Farm \\
\hline 33 & PPFM-33 & Rhizosphere & Karatagi \\
\hline 34 & PPFM-34 & Rhizosphere & Karatagi \\
\hline 35 & PPFM-35 & Phyllosphere & Karatagi \\
\hline 36 & PPFM-36 & Phyllosphere & Karatagi \\
\hline 37 & PPFM-37 & Rhizosphere & Gangavati \\
\hline 38 & PPFM-38 & Rhizosphere & Gangavati \\
\hline 39 & PPFM-39 & Phyllosphere & Gangavati \\
\hline 40 & PPFM-40 & Phyllosphere & Gangavati \\
\hline \multicolumn{4}{|c|}{ Yadgiri district } \\
\hline 41 & PPFM-41 & Rhizosphere & Hunasagi \\
\hline 42 & PPFM-42 & Rhizosphere & Hunasagi \\
\hline 43 & PPFM-43 & Phyllosphere & Hunasagi \\
\hline 44 & PPFM-44 & Phyllosphere & Hunasagi \\
\hline 45 & PPFM-45 & Rhizosphere & Shahapur \\
\hline 46 & PPFM-46 & Rhizosphere & Shahapur \\
\hline 47 & PPFM-47 & Phyllosphere & Shahapur \\
\hline 48 & PPFM-48 & Phyllosphere & Shahapur \\
\hline 49 & PPFM-49 & Rhizosphere & Balichakra \\
\hline 50 & PPFM-50 & Phyllosphere & Balichakra \\
\hline
\end{tabular}


Int.J.Curr.Microbiol.App.Sci (2019) 8(7): 152-163

Table.2 Morphological characteristics of pink pigmented facultative methylotroph isolates

\begin{tabular}{|c|c|c|c|c|c|c|}
\hline $\begin{array}{l}\text { Sl. } \\
\text { No. }\end{array}$ & Isolate code & Cell shape & Motility & Gram reaction & Pigmentation & $\begin{array}{c}\text { Accumulation of } \\
\text { polyhydroxybutyrate } \\
\text { (PHB) }\end{array}$ \\
\hline 1 & PPFM-1 & Rod & Positive & Negative & +++ & Positive \\
\hline 2 & PPFM-2 & Rod & Positive & Negative & ++ & Positive \\
\hline 3 & PPFM-3 & Rod & Positive & Negative & +++ & Positive \\
\hline 4 & PPFM -4 & Rod & Positive & Negative & +++ & Positive \\
\hline 5 & PPFM-5 & Rod & Positive & Negative & +++ & Positive \\
\hline 6 & PPFM-6 & Rod & Positive & Negative & ++ & Positive \\
\hline 7 & PPFM-7 & Rod & Positive & Negative & ++ & Positive \\
\hline 8 & PPFM-8 & Rod & Positive & Negative & +++ & Positive \\
\hline 9 & PPFM-9 & Rod & Positive & Negative & + & Positive \\
\hline 10 & PPFM-10 & Rod & Positive & Negative & ++ & Positive \\
\hline 11 & PPFM-11 & Rod & Positive & Negative & + & Positive \\
\hline 12 & PPFM-12 & Rod & Positive & Negative & ++ & Positive \\
\hline 13 & PPFM-13 & Rod & Positive & Negative & ++ & Positive \\
\hline 14 & PPFM-14 & Rod & Positive & Negative & ++ & Positive \\
\hline 15 & PPFM-15 & Rod & Positive & Negative & +++ & Positive \\
\hline 16 & PPFM-16 & Rod & Positive & Negative & +++ & Positive \\
\hline 17 & PPFM-17 & Rod & Positive & Negative & +++ & Positive \\
\hline 18 & PPFM-18 & Rod & Positive & Negative & +++ & Positive \\
\hline 19 & PPFM-19 & Rod & Positive & Negative & +++ & Positive \\
\hline 20 & PPFM-20 & Rod & Positive & Negative & + & Positive \\
\hline 21 & PPFM-21 & Rod & Positive & Negative & ++ & Positive \\
\hline 22 & PPFM-22 & Rod & Positive & Negative & +++ & Positive \\
\hline 23 & PPFM-23 & Rod & Positive & Negative & + & Positive \\
\hline 24 & PPFM-24 & Rod & Positive & Negative & ++ & Positive \\
\hline 25 & PPFM-25 & Rod & Positive & Negative & ++ & Positive \\
\hline 26 & PPFM-26 & Rod & Positive & Negative & + & Positive \\
\hline 27 & PPFM-27 & Rod & Positive & Negative & ++ & Positive \\
\hline 28 & PPFM-28 & Rod & Positive & Negative & +++ & Positive \\
\hline 29 & PPFM-29 & Rod & Positive & Negative & +++ & Positive \\
\hline 30 & PPFM-30 & Rod & Positive & Negative & +++ & Positive \\
\hline 31 & PPFM-31 & Rod & Positive & Negative & +++ & Positive \\
\hline 32 & PPFM-32 & Rod & Positive & Negative & +++ & Positive \\
\hline 33 & PPFM-33 & Rod & Positive & Negative & + & Positive \\
\hline 34 & PPFM-34 & Rod & Positive & Negative & ++ & Positive \\
\hline 35 & PPFM-35 & Rod & Positive & Negative & ++ & Positive \\
\hline 36 & PPFM-36 & Rod & Positive & Negative & + & Positive \\
\hline 37 & PPFM-37 & Rod & Positive & Negative & + & Positive \\
\hline 38 & PPFM-38 & Rod & Positive & Negative & ++ & Positive \\
\hline 39 & PPFM-39 & Rod & Positive & Negative & ++ & Positive \\
\hline 40 & PPFM-40 & Rod & Positive & Negative & ++ & Positive \\
\hline 41 & PPFM-41 & Rod & Positive & Negative & ++ & Positive \\
\hline 42 & PPFM-42 & Rod & Positive & Negative & +++ & Positive \\
\hline 43 & PPFM-43 & Rod & Positive & Negative & +++ & Positive \\
\hline 44 & PPFM-44 & Rod & Positive & Negative & +++ & Positive \\
\hline 45 & PPFM-45 & Rod & Positive & Negative & ++ & Positive \\
\hline 46 & PPFM-46 & Rod & Positive & Negative & ++ & Positive \\
\hline 47 & PPFM-47 & Rod & Positive & Negative & +++ & Positive \\
\hline 48 & PPFM-48 & Rod & Positive & Negative & + & Positive \\
\hline 49 & PPFM-49 & Rod & Positive & Negative & ++ & Positive \\
\hline 50 & PPFM-50 & Rod & Positive & Negative & ++ & Positive \\
\hline 51 & $\begin{array}{l}\text { Reference strain } \\
\text { (M. extorquens) }\end{array}$ & Rod & Positive & Negative & +++ & Positive \\
\hline
\end{tabular}

Note: + Pale pink

++ Light / Medium pink

+++ Dark / Bright pink 
Table.3 Biochemical characteristics of pink pigmented facultative methylotroph isolates

\begin{tabular}{|c|c|c|c|c|c|c|c|c|c|c|}
\hline $\begin{array}{l}\text { Sl. } \\
\text { No. }\end{array}$ & Isolates code & $\begin{array}{c}\text { Oxidase } \\
\text { Test }\end{array}$ & $\begin{array}{c}\text { Urease } \\
\text { Test }\end{array}$ & $\begin{array}{c}\text { Catalase } \\
\text { Test }\end{array}$ & $\begin{array}{c}\text { Indole } \\
\text { production } \\
\text { Test }\end{array}$ & $\begin{array}{l}\text { MR and } \\
\text { VP Test }\end{array}$ & $\begin{array}{c}\text { Citrate } \\
\text { utilization }\end{array}$ & $\begin{array}{c}\text { Casein } \\
\text { Hydrolysis }\end{array}$ & $\begin{array}{c}\text { Starch } \\
\text { Hydrolysis }\end{array}$ & $\begin{array}{c}\text { Nitrate } \\
\text { reduction } \\
\text { Test }\end{array}$ \\
\hline 1 & PPFM-1 & + & + & + & + & - & + & - & - & - \\
\hline 2 & PPFM-2 & + & + & + & + & - & + & - & - & - \\
\hline 3 & PPFM-3 & + & + & + & + & - & + & - & - & - \\
\hline 4 & PPFM -4 & + & + & + & + & - & + & - & - & - \\
\hline 5 & PPFM-5 & + & + & + & + & - & + & - & - & - \\
\hline 6 & PPFM-6 & + & + & + & + & - & + & - & - & - \\
\hline 7 & PPFM-7 & + & + & + & + & - & + & - & - & - \\
\hline 8 & PPFM-8 & + & + & + & + & - & + & - & - & - \\
\hline 9 & PPFM-9 & + & + & + & + & - & + & - & - & - \\
\hline 10 & PPFM-10 & + & + & + & + & - & + & - & - & - \\
\hline 11 & PPFM-11 & + & + & + & + & - & + & - & - & - \\
\hline 12 & PPFM-12 & + & + & + & + & - & + & - & - & - \\
\hline 13 & PPFM-13 & + & + & + & + & - & + & - & - & - \\
\hline 14 & PPFM-14 & + & + & + & + & - & + & - & - & - \\
\hline 15 & PPFM-15 & + & + & + & + & - & + & - & - & - \\
\hline 16 & PPFM-16 & + & + & + & + & - & + & - & - & - \\
\hline 17 & PPFM-17 & + & + & + & + & - & + & - & - & - \\
\hline 18 & PPFM-18 & + & + & + & + & - & + & - & - & - \\
\hline 19 & PPFM-19 & + & + & + & + & - & + & - & - & - \\
\hline 20 & PPFM-20 & + & + & + & + & - & + & - & - & - \\
\hline 21 & PPFM-21 & + & + & + & + & - & + & - & - & - \\
\hline 22 & PPFM-22 & + & + & + & + & - & + & - & - & - \\
\hline 23 & PPFM-23 & + & + & + & + & - & + & - & - & - \\
\hline 24 & PPFM-24 & + & + & + & + & - & + & - & - & - \\
\hline 25 & PPFM-25 & + & + & + & + & - & + & - & - & - \\
\hline
\end{tabular}




\begin{tabular}{|c|c|c|c|c|c|c|c|c|c|c|}
\hline 26 & PPFM-26 & + & + & + & + & - & + & - & - & - \\
\hline 27 & PPFM-27 & + & + & + & + & - & + & - & - & - \\
\hline 28 & PPFM-28 & + & + & + & + & - & + & - & - & - \\
\hline 30 & PPFM-30 & + & + & + & + & - & + & - & - & - \\
\hline 31 & PPFM-31 & + & + & + & + & - & + & - & - & - \\
\hline 32 & PPFM-32 & + & + & + & + & - & + & - & - & - \\
\hline 33 & PPFM-33 & + & + & + & + & - & + & - & - & - \\
\hline 34 & PPFM-34 & + & + & + & + & - & + & - & - & - \\
\hline 35 & PPFM-35 & + & + & + & + & - & + & - & - & - \\
\hline 36 & PPFM-36 & + & + & + & + & - & + & - & - & - \\
\hline 37 & PPFM-37 & + & + & + & + & - & + & - & - & - \\
\hline 38 & PPFM-38 & + & + & + & + & - & + & - & - & - \\
\hline 40 & PPFM-40 & + & + & + & + & - & + & - & - & - \\
\hline 41 & PPFM-41 & + & + & + & + & - & + & - & - & - \\
\hline 42 & PPFM-42 & + & + & + & + & - & + & - & - & - \\
\hline 43 & PPFM-43 & + & + & + & + & - & + & - & - & - \\
\hline 44 & PPFM-44 & + & + & + & + & - & + & - & - & - \\
\hline 45 & PPFM-45 & + & + & + & + & - & + & - & - & - \\
\hline 46 & PPFM-46 & + & + & + & + & - & + & - & - & - \\
\hline 47 & PPFM-47 & + & + & + & + & - & + & - & - & - \\
\hline 48 & PPFM-48 & + & + & + & + & - & + & - & - & - \\
\hline 49 & PPFM-49 & + & + & + & + & - & + & - & - & - \\
\hline 50 & PPFM-50 & + & + & + & + & - & + & - & - & - \\
\hline 51 & $\begin{array}{c}\text { Reference } \\
\text { strain (M. } \\
\text { extorquens) }\end{array}$ & + & + & + & + & - & + & - & - & - \\
\hline
\end{tabular}

+ Growth, - No growth 


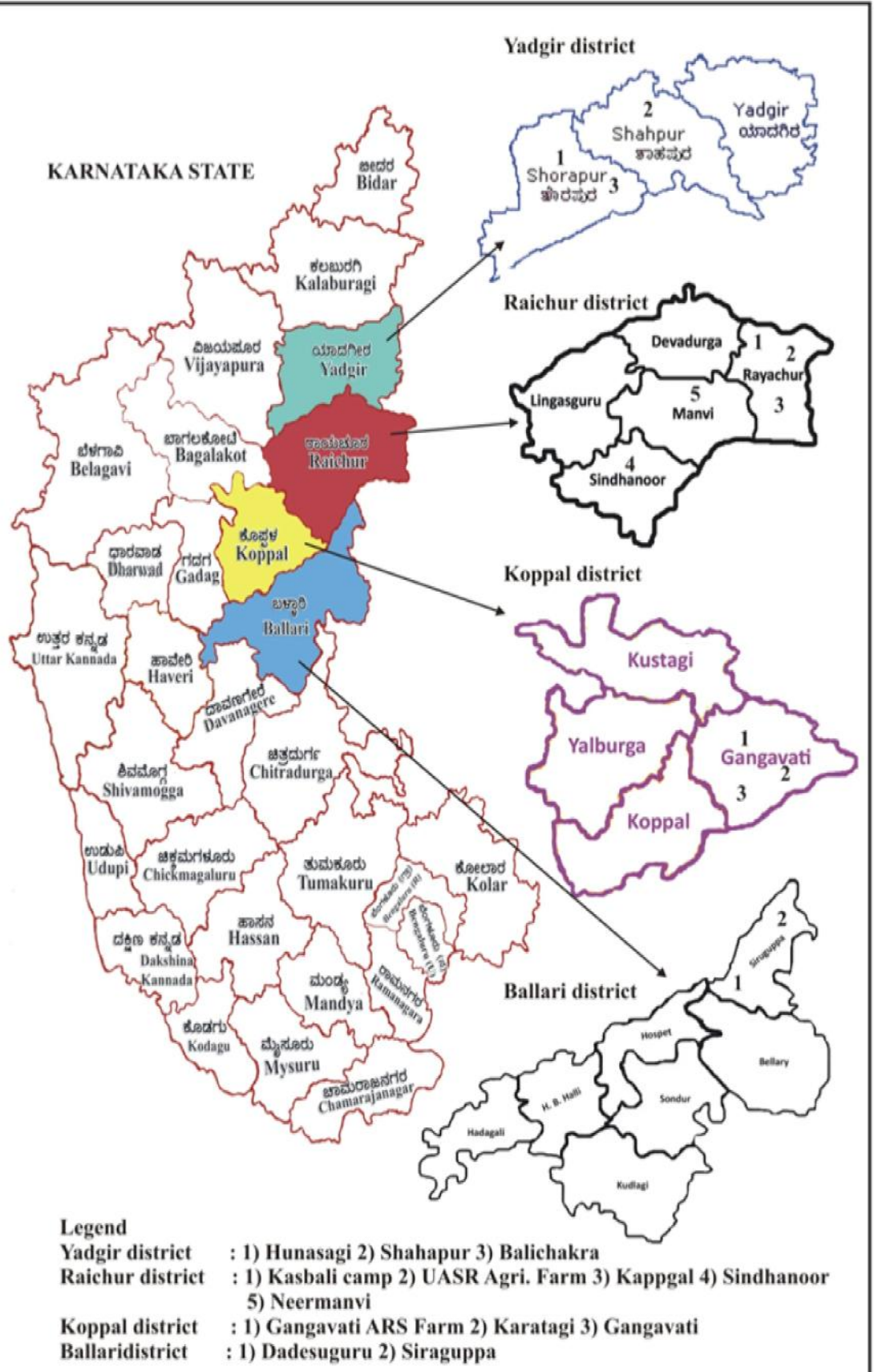

Fig. 1: Source of samples of directed seeded rice used for isolation of PPFM isolates 


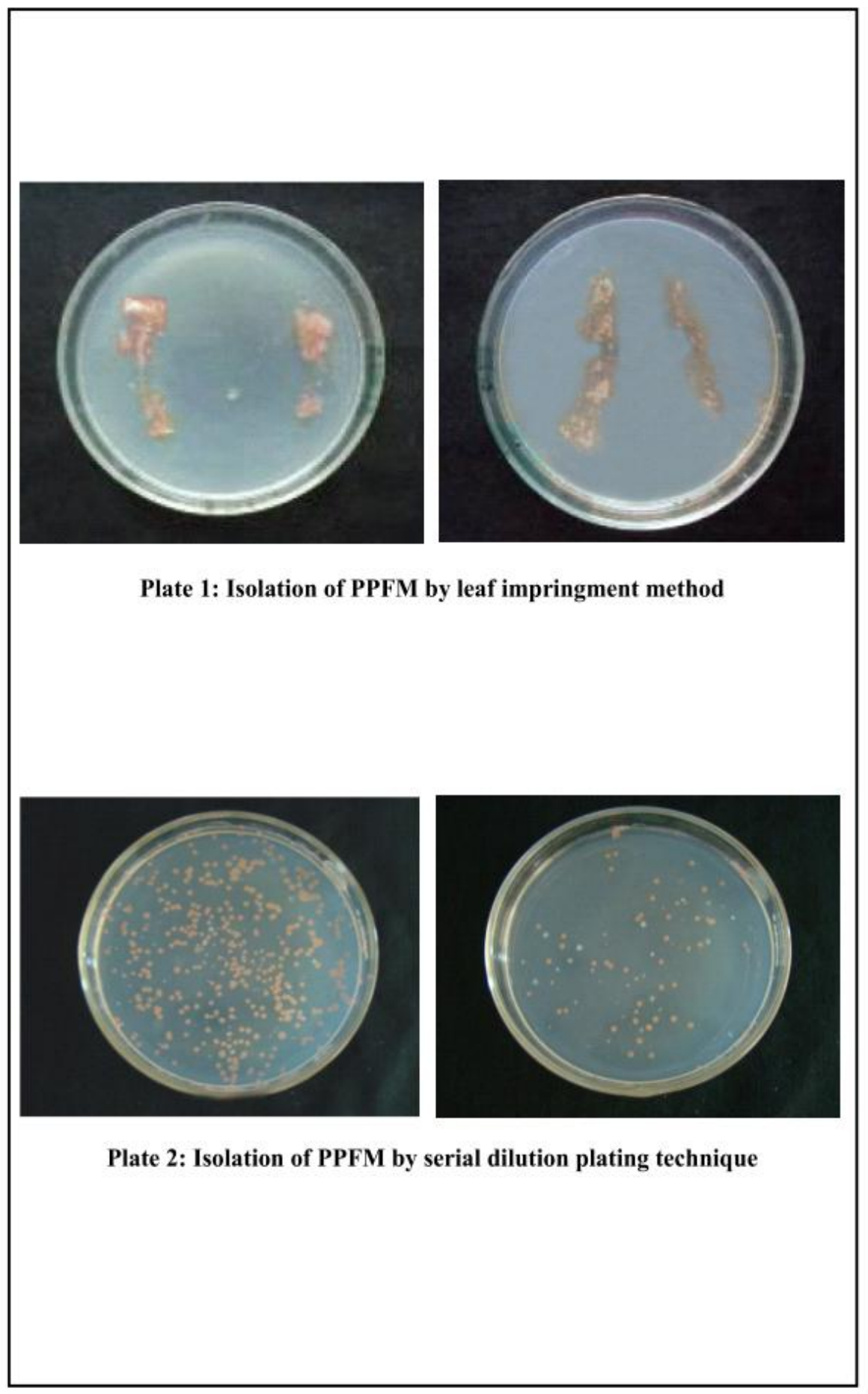




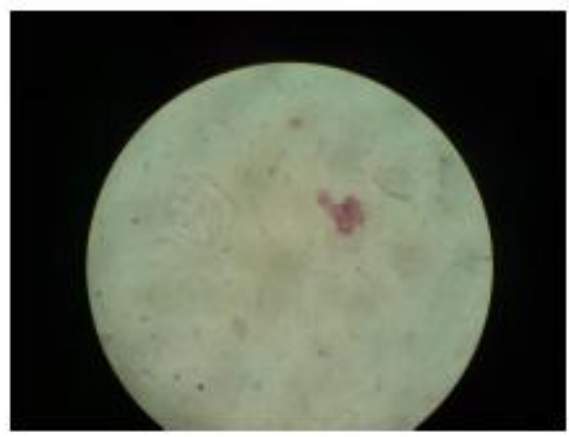

Gram staining

Biochemical characteristics of Pink pigmented facultative methylotrophs

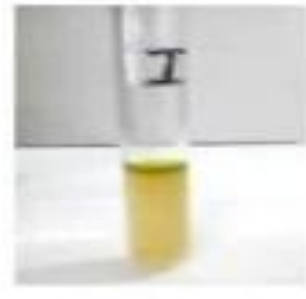

Indole Test

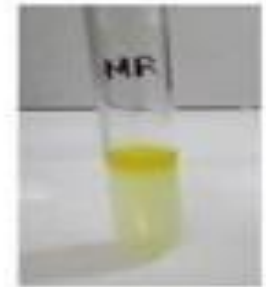

Methyl Red Test

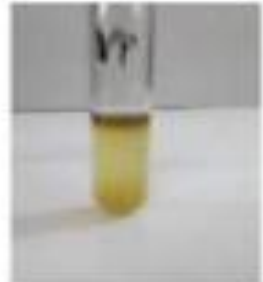

Voges Proskauer Test

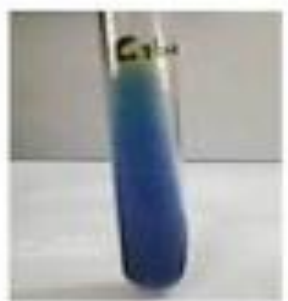

Citrate Test

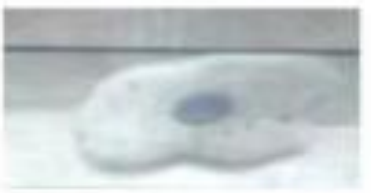

Catalase Test

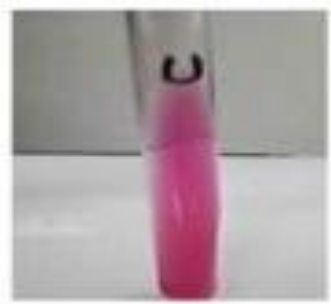

Urease Test

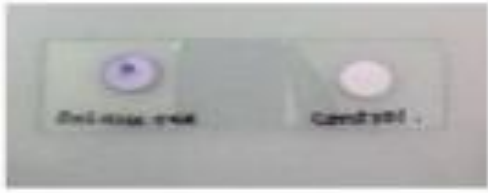

Oxidase Test
PPFM isolates were further characterized by a series of biochemical tests. All the isolates including reference strain were confirmed to be negative for Methyl red and VogesProskauer test while, positive for oxidase, urease, catalase activity, indole production and citrate utilization. None of the isolates could reduce nitrate to nitrite, whereas; the hydrolysis of casein and starch was not recorded in any of the isolates tested. Similar 
observations were reported by Thangamani (2005) and Radha et al., (2007).

\section{Summary and Conclusion}

The study demonstrated the occurrence of PPFM in the leaves AND rhizosphere region of the DSR. Based on the phenotypic characteristics of the isolates, they can be assigned to the genus Methylobacterium based on the minimum criteria set by Green (2001). Variations on the morphological and biochemical properties of the different isolates underscore the diversity of PPFM bacterial strains residing the leaves of the plant. it is interesting to note that this plant harbor PPFM bacteria. Fifty PPFMs were isolated from rhizosphere soil and phyllosphere of DSR. Out of fifty isolates, 25 were isolated from rhizosphere soil and remaining 25 isolates were isolated from phyllosphere, on AMS medium, which is a selective medium for PPFM's.

The isolates were characterized based on the morphological and biochemical characteristics. The results have clearly indicated the isolates to be positive for oxidase, urease, catalase and indole production. None of the isolates were found to be positive for casein hydrolysis, cellulose hydrolysis, nitrate reduction test, MR and VP test and starch hydrolysis. All the isolates including reference strain were Gram negative, rod shaped, exhibited motility, accumulated PHB granules with pink colonies of different color intensities.

\section{References}

Balachandra, D., Raja, P. and Sundaram, S. P., 2008, Genetic and metabolic diversity of pink-pigmented facultative Methylotrophs in phyllosphere of tropical plants, Brazilian Journal of Microbiology, 39:68-73.
Basile, D. V., Slade, L. L. and Corpe, W. A., 1969, An association between a bacterium and a liverwort, Scapania nemorosa. Bulletin of the Torrey Botanical Club, 96: 711-714.

Corpe, W. A. and Basile, D. V., 1982, Methanol-utilizing bacteria associated with green plants. Dev. Indust. Microbiol., 23: 483-493.

Corpe, W. A. and Rheem, S., 1989, Ecology of the methylotrophic bacteria on living leaf surfaces. Fems Microbiology Ecology., 62(4): 243-249.

Corpe, W. A., 1985. A method for detecting Methylotrophic bacteria on solid surfaces, J. Microbiol., 3: 483-493.

Green, P. N. and Bousfield, I. J., 1982, A taxonomic study of some gram-negative facultatively methylotrophic bacteria. Journal of General Microbiology, 128: 623-638

Green, P. N. and Bousfield, I. J., 1982, A taxonomic study of some gram-negative facultatively methylotrophic bacteria. Journal of General Microbiology, 128: 623-638

Holland, M. A., 1997, Occam's razor applied to hormonology: are cytokinins produced by plants, Plant Physiol., 115: 865-868.

Kim, K., Hwang, S., Saravanan, V. S. and Sa, T., 2010, Effect of Brevibacterium iodinum RS16 and Methylobacterium oryzae CBMB20 inoculation on seed germination and early growth of maize and sorghum-sudan grass hybrid seedling under different salinity levels. Korean J. Soil Sci. Fert., 45(1): 51-58.

Kuono, K. and Ozaki, A., 1975, Distribution and identification of methanol utilizing bacteria. In: Microbial Growth on $\mathrm{C}_{1}$ Compounds. (Eds.) Organizing committee Soc. Ferm. Tech., Japan Publisher, pp. 11-21.

Lidstrom, M. E. and Chistoserdova, L., 2002, Plants in the pink: cytokinin production 
by Methylobacterium. J. Bacteriol., 184: 1818 .

Madhaiyan, M., 2003, Molecular aspects, diversity and plant interaction of facultative methylotrophs occurring in tropical plants. Ph. D. Thesis, Tamil Nadu Agricultural University, Coimbatore.

Omer, Z. S., Tombolini, R., Broberg, A. and Gerhardson, B., 2004, Indole-3-acetic acid production by pink-pigmented facultative methylotrophic bacteria. Plant Growth Regul., 43: 93-96.

Radha, T. K., 2007, Studies on methylotrophs and their beneficial effects on soybean (Glycine $\max$ (L.) Merrill). M. Sc. (Agri.) Thesis, Univ. Agric. Sci., Dharwad (India).

Thangamani, G., 2005, Studies on facultative methylotrophs for increasing crop production. Ph. D. Thesis, Tamil Nadu Agric. Univ. Coimbatore (India).

Trotsenko, Y. A, Ivanova, E. G. and Doronina, N. V., 2001, Aerobic Methylotrophic bacteria as phytosymbionts. Microbiology, 70: 623632.

\section{How to cite this article:}

Raghavendra, J., G.P. Santhosh, Mahadevaswamy, S. Shubha and Ravi, M.V. 2019. Isolation and Identification of PPFM Bacterial Isolates Isolated From Direct Seeded Rice Growing Areas of Hyderabad-Karnataka, India. Int.J.Curr.Microbiol.App.Sci. 8(07): 152-163. doi: https://doi.org/10.20546/ijcmas.2019.807.019 